\title{
OSTEOPATIA HIPERTRÓFICA associada à metástase pulmonar de carcinoma mamário sólido grau III de malignidade em um cão - Relato de caso
}

\author{
Hypertrophic osteopathy associated with pulmonary \\ metastasis of carcinoma mammary solid grade III in a \\ dog - Case report
}

Breno Aguiar Salzedas ${ }^{1+}$, Emiliana Valloto ${ }^{2}$, Pamela Ferreira de Oliveira ${ }^{3}$, Antônio Alexandre
Speri Alves $^{4}$, Franco Ferraro Calderaro

*Autor Correspondente: Breno Aguiar Salzedas. Rua Nossa Senhora Aparecida, 232, Vila Fátima, Guarulhos - SP. CEP: 07191-190.

E-mail:breno.salzedas@gmail.com

Como citar: SALZEDAS, Breno Aguiar et al. Osteopatia hipertrófica associada à metástase pulmonar de carcinoma mamário sólido grau III de malignidade em cão - Relato de caso. Revista de Educação Continuada em Medicina Veterinária e Zootecnia do CRMV-SP, São Paulo, v.19, n. 1, 2021. e38052. Doi 10.36440/recmvz.v19i1.38052

Cite as: SALZEDAS, Breno Aguiar et al. Hypertrophic Osteopathy Associated With Pulmonary Metastasis Of Carcinoma Mammary Solid Grade III In A Dog - Case report. Journal of Continuing Education in Veterinary Medicine and Animal Science of CRMV-SP, São Paulo, v.19, n. 1, 2021. e38052. Doi 10.36440/recmvz. v19i1.38052

\section{Resumo}

A osteopatia hipertrófica é uma síndrome caracterizada por proliferação óssea em consequência de doença pulmonar, como nódulos metastáticos. 0 diagnóstico é baseado na radiografia dos ossos apendiculares evidenciando a proliferação. As neoplasias mamárias são as que mais acometem os cães e, suas variantes malignas, frequentemente metastatizam. 0 presente relato apresenta o caso de uma cadela com carcinoma mamário sólido, grau III de malignidade, que apresentou metástase pulmonar e, em decorrência, desenvolveu a osteopatia hipertrófica. Devido o estágio avançado da neoplasia, a paciente foi submetida à eutanásia. Desse modo, a osteopatia pode atuar como um sinalizador para o médico-veterinário para doenças pulmonares, sobretudo nódulos metastáticos.

Palavras-chave: Neoplasia mamária. Síndrome paraneoplásica. Canina.

1 Aprimorando de Patologia Geral e Clínica na Universidade Guarulhos, Guarulhos, SP, Brasil

2 Aprimoranda de Diagnóstico por Imagem na Universidade Guarulhos, Guarulhos, SP, Brasil

3 Aprimoranda de Patologia Geral e Clínica na Universidade Guarulhos, Guarulhos, SP, Brasil

4 Mestrando de Medicina Veterinária e Zootecnia na Faculdade de Medicina Veterinária e Zootecnia da Universidade São Paulo (FMVZ-USP), São Paulo, SP, Brasil

5 Docente de Patologia Geral na Universidade Guarulhos, Guarulhos, SP, Brasil 


\section{Abstract}

The hypertrophic osteopathy is a syndrome characterized by bone proliferation as a consequence of lung disease, such as metastatic nodules. The diagnosis is based on radiography of the appendicular bones evidencing the proliferation. Mammary neoplasms are the ones that affect dogs the most and, their malignant variants, frequently metastasize. The present report describes the case of a bitch with carcinoma mammary solid grade III of malignancy that presented pulmonary metastasis and, consequently, developed hypertrophic osteopathy. Due to advanced stage of the neoplasm the patient was submitted to euthanasia. In this way, the osteopathy can act as a signaller for the veterinarian for pulmonary diseases, especially metastatic nodules.

Keywords: Mammary neoplasm. Paraneoplasic syndrome. Canine.

\section{Introdução}

A osteopatia hipertrófica $(\mathrm{OH})$ é uma síndrome caracterizada por proliferação óssea periosteal que se manifesta, primeiramente, na diáfise dos ossos longos. (TROST et al., 2012; OLIVEIRA et al., 2013). Advém secundariamente a outras doenças, especialmente associada a afecções pulmonares crônicas ou como síndrome paraneoplásica (TROST et al., 2012; CAZZULI et al., 2017).

Apesar da patogenia da OH não ser esclarecida, sabe-se que há um aumento do fluxo sanguíneo periférico na porção distal dos membros, o que promove a proliferação de tecido conjuntivo com subsequente mineralização. Sendo assim, a osteopatia hipertrófica é caracterizada por proliferação periosteal de osso neoformado no esqueleto (CAZZULI et al., 2017).

$\mathrm{A} \mathrm{OH}$ acomete com maior frequência a superfície de ossos longos do esqueleto apendicular e normalmente os mais afetados são rádio, ulna, tíbia, metacarpo e metatarso, observando-se simetria nos quatro membros. Os ossos assemelham-se a um coral devido a sua superfície irregular e porosa (TROST et al., 2012).

A OH é uma doença mais frequentemente associada a humanos e cães (TROST et al., 2012), porém já foram descritos relatos em gatos, equinos, bovinos, primatas (TROST et al., 2012; CAZZULI et al., 2017), mustelídeos, veados, leões (TROST et al., 2012), ovinos e aves domésticas (CAZZULI et al., 2017). Dentre os cães, a incidência varia de um a 15 anos, sendo àqueles da raça Boxer os mais acometidos.

A OH paraneoplásica está associada a neoplasias pulmonares, sejam estas de origem primária ou metastática (OLIVEIRA et al., 2013).

O paciente acometido pela $\mathrm{OH}$ apresenta dor, aumento de volume simétrico e (MENEZES et al., 2012), hipertermia nas extremidades dos ossos envolvidos, resultando em claudicação alternada, relutância em se movimentar (OLIVEIRA et al., 2013) e rigidez muscular (CAZZULI et al., 2017). Pacientes com quadros clínicos mais avançados podem exibir extremidades ósseas deformadas ou espessadas. Por ser uma doença relacionada a neoplasias pulmonares, também são achados sinais clínicos como dispneia, tosse e sons pulmonares alterados à auscultação (OLIVEIRA et al., 2013).

$\mathrm{O}$ diagnóstico da $\mathrm{OH}$ baseia-se no exame radiográfico simples do esqueleto apendicular dos animais em que se evidencia neoformação óssea periosteal distribuída simetricamente e uniforme ao longo das diáfises dos ossos longos e das falanges, sendo os ossos do carpo e do tarso menos gravemente afetados, sem envolvimento articular (KROLIKOWSKI, 2006; RAHAL, et.al., 2003).

Otratamento da $\mathrm{OH}$ é inespecífico, baseando-se na remoção da causa primária o que, por consequência, induz a regressão das manifestações clínicas e, parcialmente, das lesões periosteais (HERMETO et al., 2013).

As neoplasias mamárias são responsáveis por mais de $50 \%$ dos casos tumorais em cadelas, sendo aquelas com média de dez anos as mais acometidas (CALDAS et al., 2016). Os carcinomas mamários basicamente são classificados de acordo com sua arquitetura, como tubulares, túbulo papilíferos e sólidos, e graduados conforme a porcentagem de estruturas tubulares encontradas, pleomorfismo 
nuclear e número de mitoses por dez campos de maior aumento, gerando uma pontuação que classifica os carcinomas entre I (bem diferenciado), II (moderadamente diferenciado) ou III (mal diferenciado). As metástases são frequentes em neoplasias mamárias malignas, sendo os linfonodos, pulmões, coração, baço, adrenais e encéfalo as regiões anatômicas mais frequentes (CALDAS et al., 2016). Sendo que, as metástases pulmonares podem estar associadas a OH (OLIVEIRA et al., 2013).

$\mathrm{O}$ presente relato tem como objetivo descrever o diagnóstico por imagem da $\mathrm{OH}$ em uma cadela, analisando as alterações patológicas encontradas macroscopicamente e microscopicamente a partir da eutanásia, ressaltando a importância do conhecimento de tal síndrome e do tratamento precoce de neoplasias associadas.

\section{Relato de caso}

Um cão SRD, 12 anos de idade, fêmea foi levado à Clínica Escola da Universidade Guarulhos (UnG) com queixa de aumento de volume de todos os membros há um mês, impotência funcional dos membros pélvicos há duas semanas, anorexia e adipsia há dois dias e em estado epilético por cerca de quatro horas. Após controle dos episódios convulsivos constatou-se, no exame físico, caquexia com aumento de volume não edematoso, hipertermia e sensibilidade dolorosa nos quatro membros e duas neoformações em cadeia mamária, M5 esquerda e M2 direita, ulceradas, que os tutores não souberam informar quando houve o surgimento e a progressão, além de apresentar desidratação, taquicardia, taquipneia, mucosas hipocoradas, sialorreia e diarreia.

Foram realizados exames radiográficos nos membros torácicos (Figura 1), observandose presença de reação periosteal proliferativa junto às regiões de diáfise medial e distal, além de epífise distal de úmero, bilateral e reação periosteal proliferativa junto às regiões de diáfise proximal, médio e distal, assim como nas epífises proximal e distal de rádio e ulna, bilaterais.

Figura 1: Radiografia do membro torácico direito de cadela com proliferação óssea

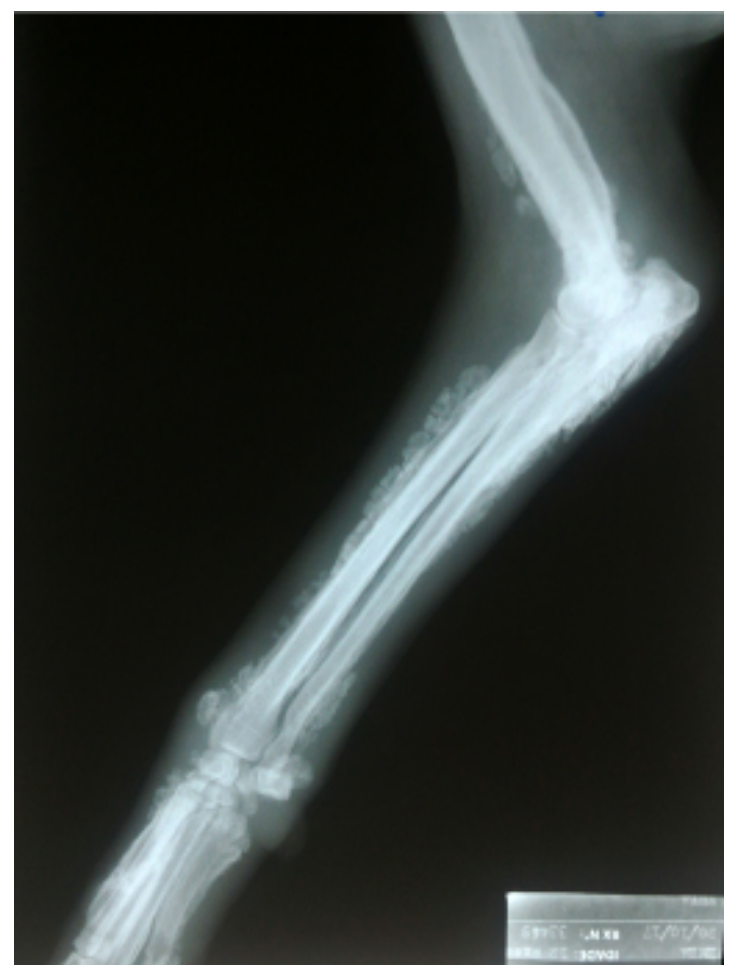

TFonte: Os autores (2020). 
Também foi realizado o exame radiográfico do tórax da paciente (Figura 2), notando-se a presença de nódulos dispersos pelo parênquima pulmonar, de contornos regulares, radiopacidade água, com dimensões entre $0,8 \mathrm{~cm} \times 0,9 \mathrm{~cm}$ a $1,7 \mathrm{~cm} \times 1,5 \mathrm{~cm}$ em seus maiores eixos. Tais estruturas condizem com nódulos metastáticos.

Figura 2: Radiografia de tórax de cadela, condizente com nódulos metastáticos em pulmão

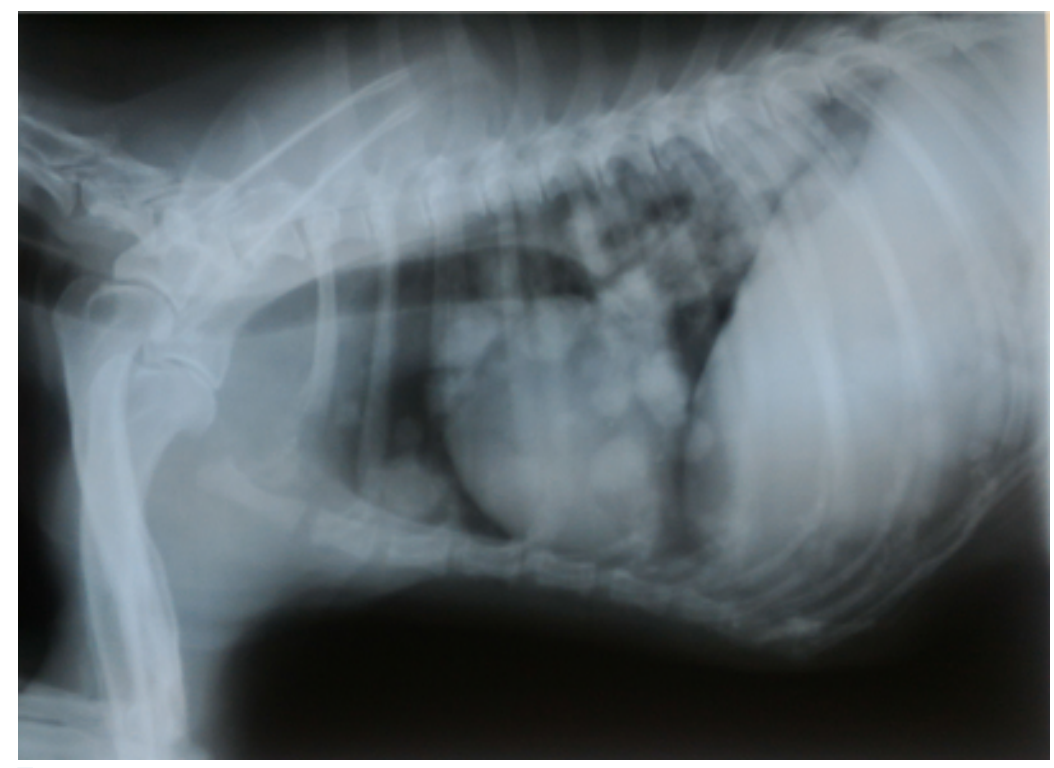

TFonte: Os autores (2020).

Diante da comorbidade em que o paciente se encontrava, seus tutores optaram pela realização da eutanásia.

Ao exame necroscópico notou-se, principalmente ao exame externo, aumento de volume nos quatro membros do paciente (gura 3) e duas neoformações mamárias (Figura 4).

Figura 3: Cão fêmea, com aumento de volume em membros e neoplasia mamária

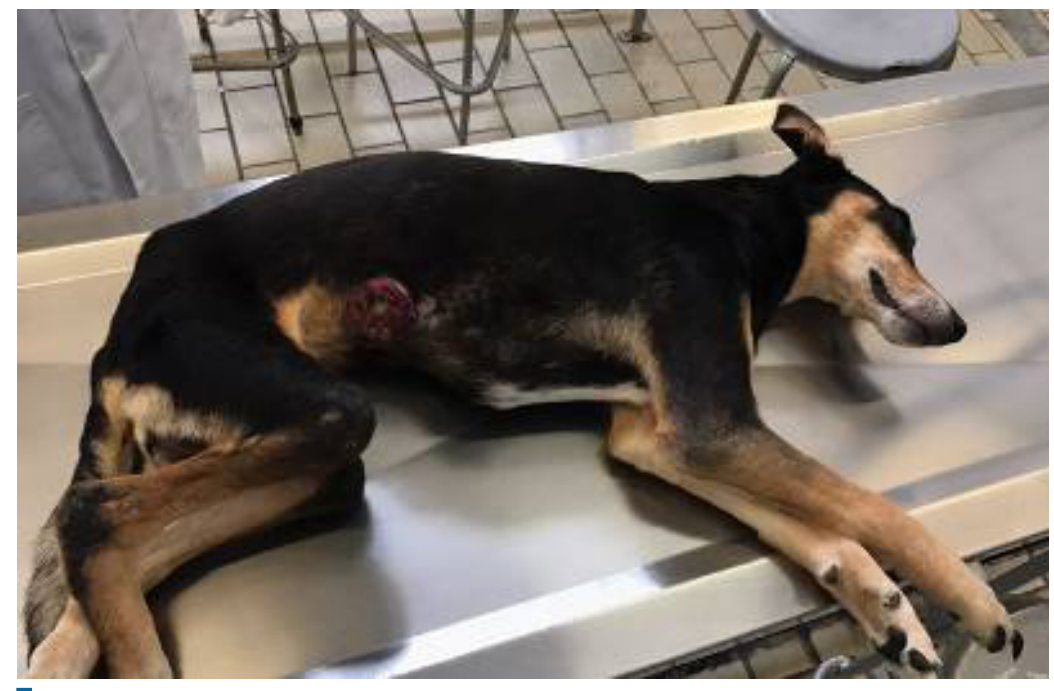

T Fonte: Os autores (2020). 
Figura 4: Cão fêmea, com neoplasias mamárias ulceradas nas glândulas mamárias abdominal cranial direita e inguinal esquerda

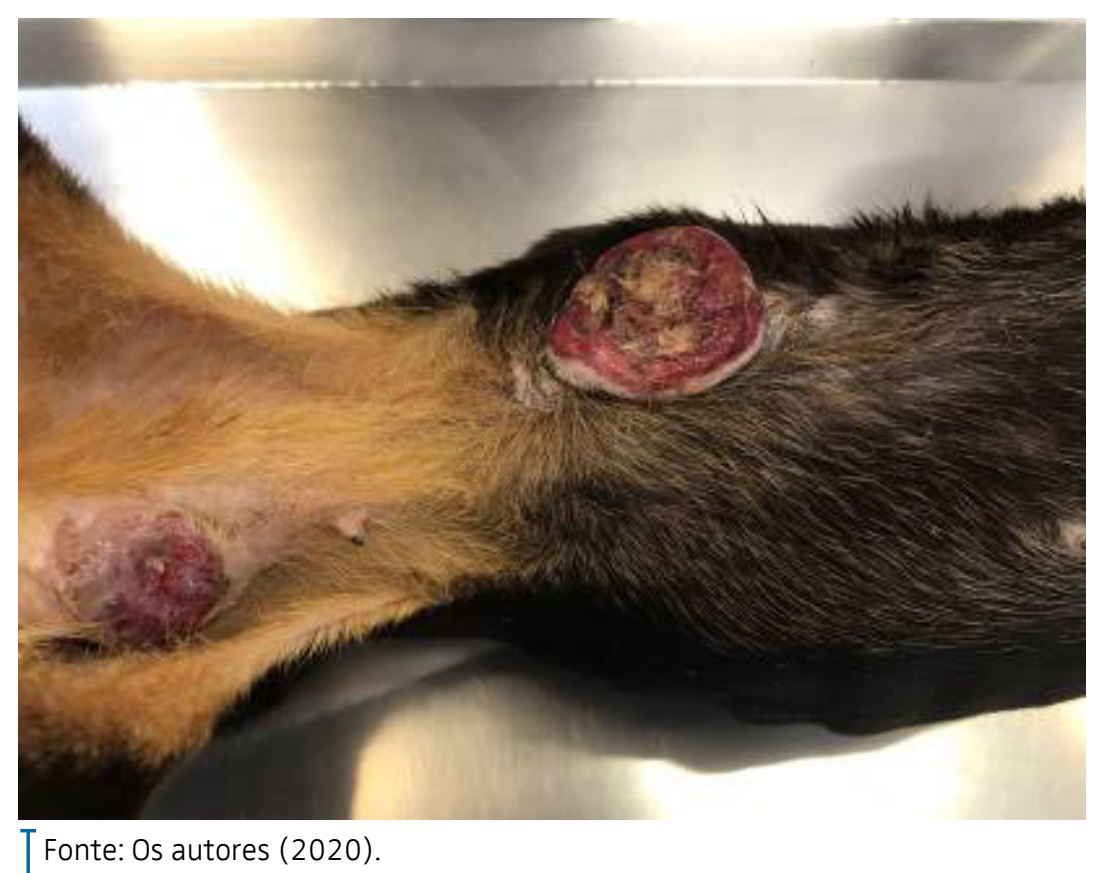

Com relação às vísceras internas, observou-se em todos os lobos pulmonares, diversas neoformações nodulares de coloração esbranquiçada, consistência firme e tamanhos variáveis, entre $0,8 \mathrm{~cm} \times 0,9 \mathrm{~cm}$ a $1,7 \mathrm{~cm} \times 1,5 \mathrm{~cm}$ (Figura 5). Além disso, os pulmões apresentaram coloração escura avermelhada multifocal, abaulamento dos lobos e presença de material espumoso ao corte caracterizando congestão moderada e edema pulmonar. No coração havia espessamento valvar condizente com endocardiose, o fígado apresentava os lobos abaulados e de coloração vermelha escura de forma difusa compatível com congestão acentuada. Também foi observada acentuada congestão em ambos os rins e congestão moderada em baço.

Figura 5: Nódulos metastáticos em pulmão de cadela

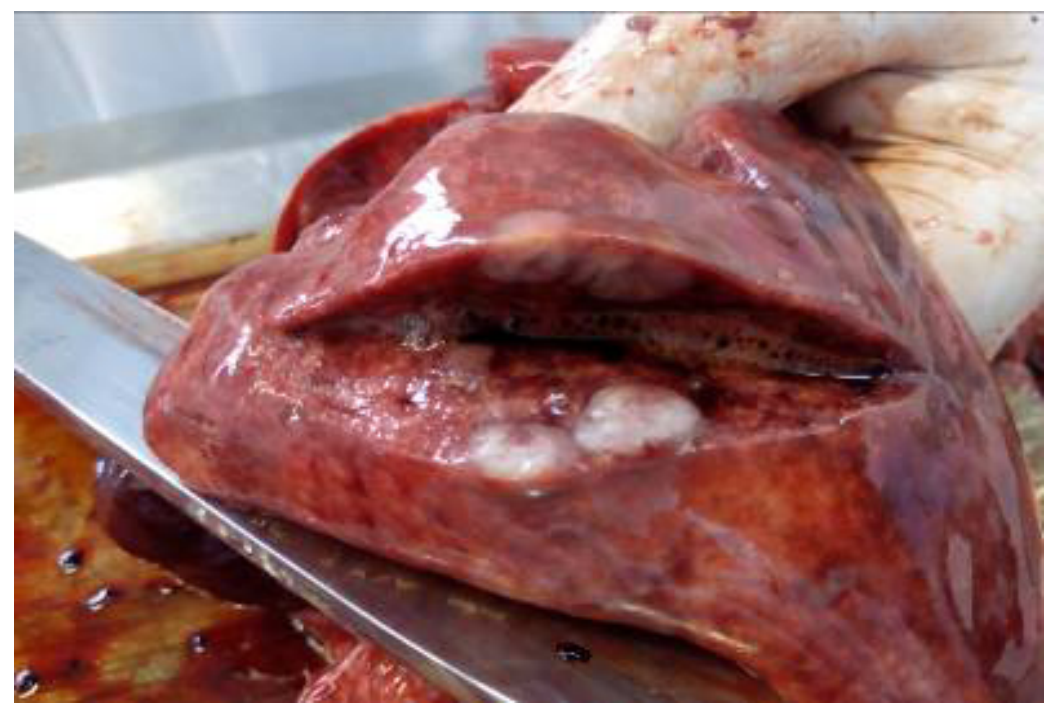

TFonte: Os autores (2020). 
A forma de apresentação clínica da doença mais grave é a neuroinvasiva, com acometimento Os ossos dos membros torácicos e pélvicos direitos e esquerdos tinham aparência porosa e irregular, assemelhando-se a corais. Os ossos mais acometidos eram a escápula, úmero e rádio de ambos os membros (Figura 6).

Figura 6: Ossos de cadela acometidos pela osteopatia hipertrófica. Método: maceração biológica

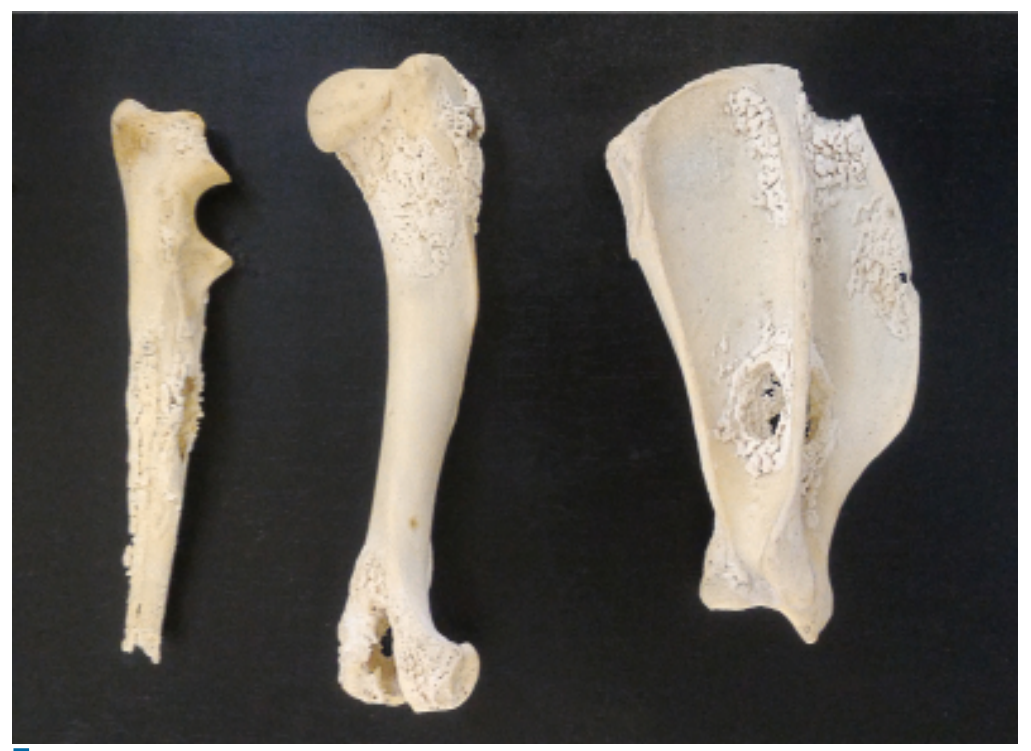

T Fonte: Os autores (2020).

No exame histopatológico das neoformações mamárias foi observada a presença de estruturas sólidas compostas por células poligonais com núcleo redondo a oval, cromatina frouxa e nucléolos proeminentes. A anisocitose, a anisocariose e o pleomorfismo eram acentuados e havia mais de dez mitoses típicas e atípicas em dez campos de maior aumento. Os vasos sanguíneos estromais estavam repletos de células tumorais caracterizando êmbolos neoplásicos. 0 diagnóstico foi dado como carcinoma mamário sólido grau III de malignidade de acordo com a classificação e graduação de tumores mamários caninos (Figura 7).

Figura 7: Carcinoma mamário sólido em cadela. Coloração: H\&E. Aumento: 400x

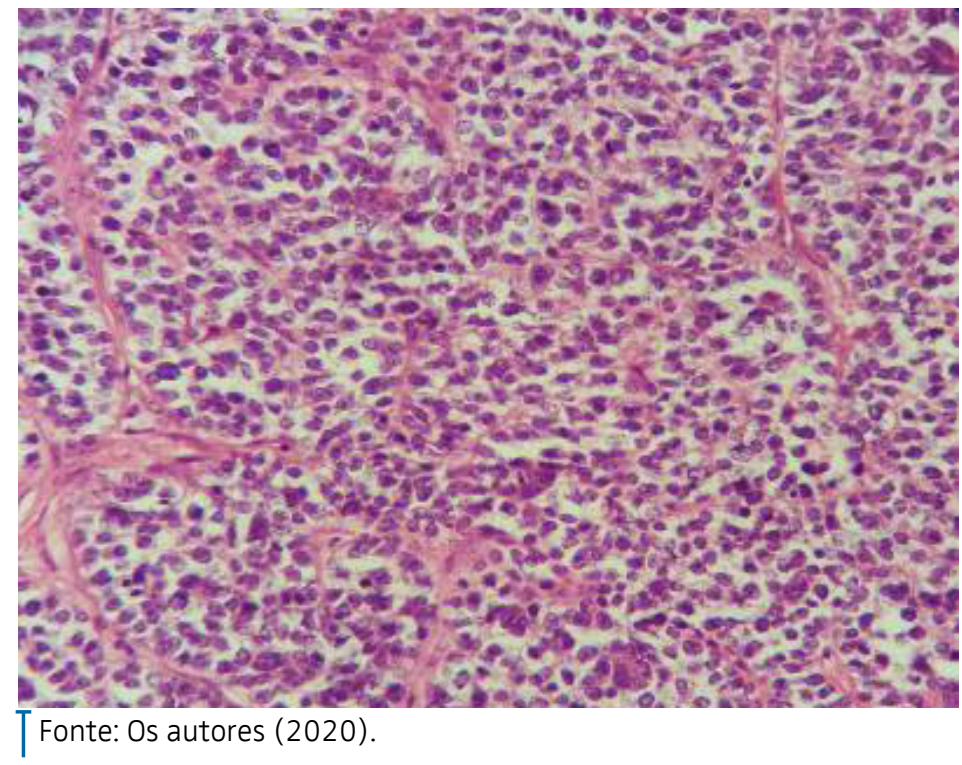


No exame histopatológico dos pulmões da cadela do caso descrito notaram-se áreas delimitadas por uma camada de células fusiformes formando um túbulo apresentando, no interior, células poligonais agrupadas de aspecto semelhante ao da neoplasia mamária, o que condiz com focos metastáticos.

\section{Discussão}

Dentre todas as neoplasias caninas, as da glândula mamária são as mais observadas em fêmeas com idade média entre 10 (CALDAS et al., 2016) e 10,6 anos (PINTO, 2009) ou mais idosas, assim como na cadela enferma do presente caso, contudo, pacientes com raça indefinida são menos predispostos a tais neoplasias (PINTO, 2009).

Devido ao desenvolvimento acelerado e progressivo das neoplasias mamárias em cadelas tanto quanto a demora por parte dos tutores em consultar um médico veterinário, é comum que os pacientes apresentem duas ou mais nodulações mamárias (PINTO, 2009; CALDAS et al., 2016), tal afirmação condiz com o presente estudo, visto que a impotência funcional dos membros do animal do caso descrito foi o que incentivou o tutor a consultar um veterinário, ao invés do surgimento das neoplasias.

O diagnóstico de carcinoma sólido pulmonar é apontado como um dos mais dificilmente constatados, chegando a 4,2\% (NEVES, 2018). A metastatização à distância é relatada como mais comum em pulmão (60\%) (NEVES, 2018), porém há controvérsias entre a frequência de metastatização de neoplasias mamárias em que Pinto (2009) relata em torno de 15\% e Neves (2018), 38,4\%. Com exceção do diagnóstico, a paciente do caso descrito apresentou todas as características mais frequentes com relação à neoplasia mamária e a soma de fatores como idade avançada, diagnóstico tardio de carcinoma com uma graduação alta e a presença de metástase, aspectos indicativos de um mau prognóstico.

Por ser considerada uma síndrome paraneoplásica e possuir relação com doenças pulmonares, a $\mathrm{OH}$ do presente caso provavelmente se desenvolveu como consequência da metástase pulmonar do carcinoma mamário, já que a doença neoplásica se apresentava em estágio avançado, corroborando com o surgimento da afecção óssea. Dados como idade e sexo não são relevantes na discussão, pois é variável a idade de acometimento e não há predileção sexual (GOMES et al., 2017). No entanto, alguns autores referem-se à predileção racial (KROLIKOWSKI, 2006), enquanto outros não a notam (GOMES et al., 2017).

As manifestações clínicas observadas no presente caso são comumente encontradas na $\mathrm{OH}$ (RAHAL et al., 2003; HERMETO et al., 2013; KROLIKOWKISKI, 2006), entretanto, de acordo com a literatura consultada, apenas o caso relatado apresentou sinais neurológicos.

Apesar da osteopatia hipertrófica ser descrita radiograficamente de forma idêntica em todos os trabalhos articular (RAHAL et al., 2003; KROLIKOWKISKI, 2006; HERMETO et al., 2013; , também relataram o aumento de radiopacidade de tecidos moles, o que não foi visualizado na paciente. Os ossos acometidos seguiram o padrão descrito, principalmente na diáfise dos ossos distais de membros e sem o envolvimento articular (RAHAL et al., 2003; KROLIKOWKISKI, 2006; MENEZES et al., 2012; HERMETO et al., 2013; HOFFMAN et al., 2017). O acometimento de epífise e de ossos proximais de membros, constatado no presente estudo.

A remoção da causa primária pode levar a regressão da proliferação óssea e da manifestação clínica (HARA et al., 1994) contudo, no presente caso, a paciente não foi tratada devido ao estado avançado em que a neoplasia mamária se encontrava. 


\section{Considerações finais}

O prévio diagnóstico de neoplasias de qualquer gênero e o tratamento precoce reduzem os riscos de metástases nos pulmões, o que, por consequência, reduz a possibilidade do aparecimento da osteopatia hipertrófica. Contudo, o aparecimento da osteopatia hipertrófica pode ser um sinalizador de doença pulmonar metastática, sendo que a sobrevida do paciente está diretamente relacionada à classificação do tumor e o seu prognóstico.\&

\section{Referências}

CALDAS, S.L. et al. Aspectos clínico-patológicos das neoplasias mamárias em cadelas (Canis familiaris). Revista Brasileira de Medicina Veterinária. Rio de Janeiro, v. 38, n. 2, p. 81-85, 2016.

CAZZULI, G. et al. Osteopatía hipertrófica canina secundaria a enfermedad extratorácica (carcinoma de células transicionales): reporte de caso. Veterinaria (Montev.). Montevideo, v. 54, n. 207, p. 14-22, 2017.

GOMES, L. G. et al. Osteopatia hipertrófica associada à metástase intratorácica de tumor mamário canino: Relato de dois casos. Veterinária e Zootecnia, v. 24, n. 2, p. 296-302, 2017. DOl: https://doi. org/10.35172/rvz.2017.v24.302.

HARA, Y. et al. Regression of hypertrophic osteopathy following removal of intrathoracic neoplasia derived from vagus nerve in a dog. Journal of Veterinary Medical Science, v. 57, n. 1, p. 133-135, 1995. DOI: https://doi.org/10.1292/jvms.57.133.

HERMETO, L. C. et al. Osteopatia hipertrófica pulmonar: alterações clínicas e radiográficas em um paciente canino. Arquives of Veterinary Sciece, Jaboticabal, v. 18, n. 2, p. 50-55, 2013. DOI: http:// dx.doi.org/10.5380/avs.v18i2.27143.

KROLIKOWSKI, G. Osteopatia hipertrófica em cães. 2006. Dissertação (Especialização em Clínica Médica e Cirúrgica de Pequenos Animais) - Universidade Federal de Santa Maria Centro de Ciências Rurais, Santa Maria, 2006.

NEVES, J. M. S. R. Neoplasias mamárias em cadelas: estudo descritivo de 29 casos clínicos. 2018. Dissertação - Faculdade de Medicina Veterinária, Universidade Lusófona de Humanidades e Tecnologias, Lisboa, 2018.

OLIVEIRA, K. M. et al. Principais síndromes paraneoplásicas em cães e gatos. Encicl. Biosfera. Goiânia, v. 9, n. 17, p. 2073-2088, 2013.

PINTO, R. M. M. O. Neoplasia mamária em cadelas e gatas. 2009. Dissertação (Mestrado Integrado de Medicina Veterinária) - Faculdade de Medicina Veterinária, Universidade Técnica de Lisboa, Lisboa, 2009.

RAHAL, S.C.; MAMPRIM, M.J.; SEQUEIRA, J.L.; FRANCO, J.P.R. Osteopatia hipertrófica associada à metástase pulmonar de osteossarcoma em um cão. Ars Veterinária, Jaboticabal, v. 19, n. 1, p. 035 - 039, 2003.

SOAVE, T. et al. A importância do exame radiográfico torácico na abordagem de animais portadores de neoplasias. Semina: Ciências Agrárias, Londrina, v.29, n.2, p. 399-406, 2008. DOl: http://dx.doi. org/10.5433/1679-0359.2008v29n2p399.

TROST, M. E. et al. A. Osteopatia hipertrófica em sete cães. Pesquisa Veterinária Brasileira. Rio de Janeiro, v. 32, n. 5, p. 424-429, 2012. DOl: https://doi.org/10.1590/S0100-736X2012000500011. 